CLINICAL STUDY

\title{
Nocturnal calcium, phosphorus and parathyroid hormone in the diagnosis of concealed and subclinical hypoparathyroidism
}

\author{
Leah Even ${ }^{1,3}$, Tarif Bader ${ }^{1}$ and Ze'ev Hochberg ${ }^{2,3}$ \\ ${ }^{1}$ Department of Pediatrics, Nahariya Hospital, Nahariya, Israel, ${ }^{2}$ Division of Pediatric Endocrinology, Rambam Medical Center, Meyer Children's Hospital, \\ PO Box 9602, Haifa 31096, Israel and ${ }^{3}$ Faculty of Medicine, Technion, Israel Institute of Technology, Haifa, Israel
}

(Correspondence should be addressed to Z Hochberg who is now at Division of Pediatric Endocrinology, Rambam Medical Center, Meyer Children's Hospital, PO Box 9602, Haifa 31096, Israel; Email: z_hochberg@rambam.health.gov.il)

\begin{abstract}
Context: Circadian rhythms of plasma parathyroid hormone (PTH) show peak values at night, whereas serum calcium levels peak in the evening and display a nadir at night.

Hypotheses: Subclinical hypoparathyroidism (HPT) can be detected by utilizing the knowledge of diurnal variations. Thalassemia major (TM) may provide a model system of subclinical HPT.

Design: Nocturnal plasma PTH and serum calcium values were determined in 13 TM patients with normal morning serum calcium levels as compared with the corresponding values in eight healthy control subjects.

Results: Six patients with TM presented a nadir serum calcium level of $8.3 \mathrm{mg} / \mathrm{dl}$ or lower (hypoCa $\mathrm{TM}$ ) at $0200 \mathrm{~h}$, whereas the remaining seven showed nadir levels of $8.4 \mathrm{mg} / \mathrm{dl}$ or higher (normoCa TM). Patients with hypoCa TM displayed a drop between peak and nadir of $1.2 \pm 0.5 \mathrm{mg} / \mathrm{dl}$ as compared with a considerably smaller fall of $0.3 \pm 0.7 \mathrm{mg} / \mathrm{dl}$ in control subjects $(P<0.05)$. NormoCa TM patients experienced comparable nocturnal variation to that of control subjects. Patients from both the hypoCa and normoCa TM groups presented significantly lower nocturnal PTH levels than those of control subjects and lost the nocturnal PTH variation characteristic of healthy subjects. A plot of all serum calcium against plasma PTH levels provides a clear distinction of the three groups.

Conclusions: All 13 daytime normocalcemic TM patients presented a certain degree of HPT. The hypoCa TM group displayed a concealed HPT detected in all, except the morning sampling, whereas normoCa TM patients experienced sub clinical HPT observed in the absence of nocturnal HPT variation. Nocturnal measurements of serum minerals thus enhance the sensitivity of HPT diagnosis.
\end{abstract}

European Journal of Endocrinology 156 113-116

\section{Introduction}

In clinical practice, the diagnosis of hypoparathyroidism (HPT) is based on measuring the morning values of serum calcium, phosphorus and plasma parathyroid hormone (PTH), without their diurnal variation. However, circadian variations of serum calcium and PTH have been reported, with peak values of PTH between 0200 and $0400 \mathrm{~h}$, whereas serum calcium levels peak at $2000 \mathrm{~h}$, then decreasing progressively to reach the lowest levels between 0200 and $0400 \mathrm{~h}$ (1). Moreover, serum levels of calcium and phosphorus are age dependent, unlike the circadian variation of calcium and PTH, which do not vary with age. Rather, this variation reflects endogenous circadian rhythms, which persist during both sleep and wakefulness conditions (1).

The working hypothesis for the present study was that subclinical HPT should be detectable, calculating the diurnal variations based on nocturnal sampling, and that it initially would manifest as reduced PTH levels at the expected hour of its peak level, accompanied by low calcium levels at the hour of its nadir levels.

The model system we used to study subclinical HPT was the endocrinopathy of thalassemia major (TM). This syndrome may include growth retardation, delayed puberty and infertility, osteoporosis, diabetes mellitus, hypothyroidism, hypoadrenalism, and HPT. The latter was reported in as many as $4.5-30 \%$ of TM patients (2-6). We predicted that prior to the development of a full-blown HPT, TM patients might experience a subclinical disease, which we defined according to the $0800 \mathrm{~h}$ serum calcium levels as normocalcemic HPT. Our findings support the notion that multiple daily measurements of PTH and calcium levels, combined with the knowledge of characteristic diurnal variation in these levels, should enable reliable detection and classification of subclinical HPT. 


\section{Patients and methods}

The Nahariya Hospital Internal Review Board approved the study protocol, and both patients and parents signed informed consents. Thirteen TM patients, age $17 \pm 3.1$ (mean \pm s.D.) years and Tanner stage 1-4, were recruited for this study. All of them presented with normal morning levels of serum calcium, as defined by the adolescence lowest normal value of $8.4 \mathrm{mg} / \mathrm{dl}$ $(2.1 \mathrm{mmol} / \mathrm{l})(7)$. Eight sex- and Tanner stage-matched healthy subjects served as controls. Tanner stage matching was employed as an additional measure because mineral metabolism correlates better with pubertal development than age $(7,8)$. All TM patients were regularly treated by transfusion of packed red cells every three weeks (at a pre-transfusion hemoglobin level of $7.8-10.2 \mathrm{~g} / \mathrm{dl}$ ), and received daily chelating therapy with s.c. desferrioxamine mesylate (Desferal, Teva, Israel). Patients did not receive calcium or vitamin D supplements.

Subjects were admitted at afternoon hours, 3 weeks after the last blood transfusion, to eliminate the effect of citrate on calcium level. After a light non-dairy supper at $1800 \mathrm{~h}$, serum calcium, phosphorus, creatinine and plasma PTH were taken at four time points $(2000,0000$ (midnight), 0200, and $0800 \mathrm{~h}$ ). $25 \mathrm{OH}-\mathrm{Vit} \mathrm{D}, 1,25$ dihydroxyvitamin $\mathrm{D}$, ferittin and hemoglobin levels were taken at $2000 \mathrm{~h}$ as baseline.

Plasma PTH was measured by IRMA for intact hPTH1-84 (PTH-S; N-tact PTH SP IRMA kit DiaSorin, Stillwater, MN, USA). Serum calcium and phosphorus were measured by the Aeroset system and the Architect c8000 system (Abbott Laboratories, Abbott Park, IL, USA). Serum calcium levels of $8.4-10.2 \mathrm{mg} / \mathrm{dl}(2.1-$ $2.55 \mathrm{mmol} / \mathrm{l})(7)$ and serum phosphorus levels of 3.6$5.7 \mathrm{mg} / \mathrm{dl}(1.2-1.8 \mathrm{mmol} / \mathrm{l})$ were considered normal range for adolescents.

Data are reported as mean \pm s.D., the three groups and the four time points were compared by the Wilcoxon rank-sum test and multivariate test.

\section{Results}

The nocturnal serum calcium levels in the control group corresponded to previous reports of peak levels at $2000 \mathrm{~h}$ decreasing to nadir at $0200 \mathrm{~h}$ and recovered at $0800 \mathrm{~h}$ (Fig. 1). Among patients with TM, five showed $0200 \mathrm{~h}$ nadirs of $8.3 \mathrm{mg} / \mathrm{dl}$ or lower $(6.8-8.3 \mathrm{mg} / \mathrm{dl}$, $1.7-2.0 \mathrm{mmol} / \mathrm{l})$, and were designated hypocalcemic (hypoCa) TM, the remaining seven patients showed nadir calcium levels over $8.3 \mathrm{mg} / \mathrm{dl}(8.4-9.2 \mathrm{mg} / \mathrm{dl}$, 2.1-2.3 mmol/l), and were designated normoCa TM (Table 1). The former group was older (mean 19.4 years) than the latter (16.3 years). HypoCa TM patients presented a decrease between peak and nadir of $1.2 \pm$ $0.5 \mathrm{mg} / \mathrm{dl}$ when compared with a decline of $0.3 \pm$ $0.7 \mathrm{mg} / \mathrm{dl}$ in control subjects $(P<0.05)$. NormoCa TM

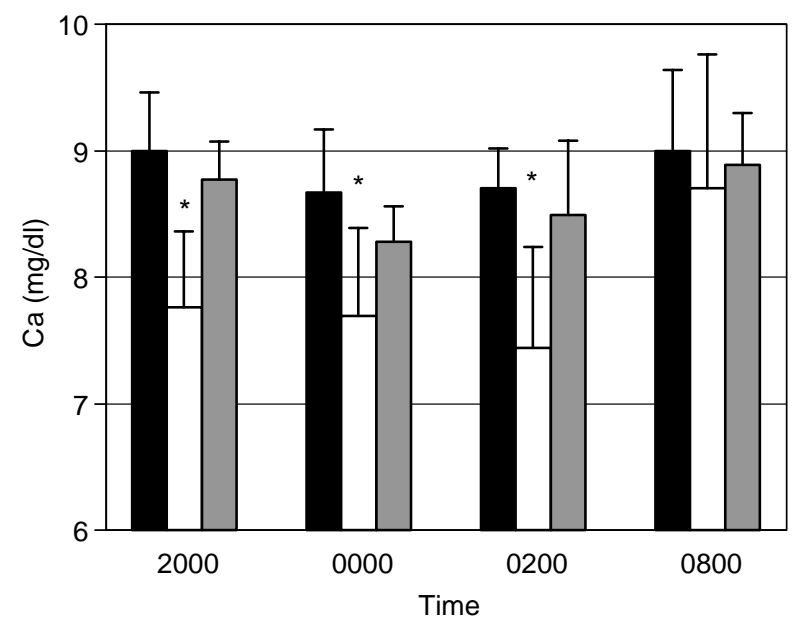

Figure 1 Nocturnal pattern of serum calcium $(\mathrm{Ca})(\mathrm{mg} / \mathrm{dl})$ in healthy controls (black bars), hypocalcemic thalassemia major (TM) (white bars) and normocalcemic TM patients (gray bars). Mean \pm s.D., ${ }^{\star} P<0.05$.

patients displayed comparable nocturnal variation to that observed in controls.

The nocturnal serum phosphorus levels of the control group showed a mirror image of serum calcium levels, with rising values from $2000 \mathrm{~h}$ through $0200 \mathrm{~h}$, receding by $0800 \mathrm{~h}$ (Fig. 2). The hypoCa TM group presented higher phosphorus levels at all time points $(P<0.05)$, whereas the NormoCa TM group showed normal phosphorus levels, as compared with control subjects. The nocturnal variations did not reach statistical significance for any of the groups.

Control subjects showed predictably nocturnal peak levels of plasma PTH (Fig. 3). The hypoCa and normoCa TM groups presented significantly lower PTH levels than those of control subjects at all time points. Importantly, patients from both groups lost the nocturnal variation, characteristic of control subjects (ANOVA $P<0.05$ ).

Dot plot of all serum calcium levels as a function of plasma PTH levels shows distinct distribution in each of the three groups examined (Fig. 4) with significant differences (multivariate test $P<0.001$ ).

Table 1 Characteristics of the study subjects.

\begin{tabular}{lccc}
\hline & Control & HypoCa TM & NormoCa TM \\
\hline $\mathrm{N}$ & 8 & 6 & 7 \\
Age (year) & $12.6 \pm 1.8$ & $19.4 \pm 5.2$ & $16.3 \pm 4.7$ \\
Sex F/M & $3 / 5$ & $3 / 2$ & $3 / 4$ \\
$\mathrm{Hb}(\mathrm{mg} \%)$ & $12.6 \pm 0.5$ & $8.0 \pm 0.3$ & $8.8 \pm 0.5$ \\
$\mathrm{Ferritin}(\mathrm{ng} / \mathrm{ml})$ & $34 \pm 13.9 ;$ & $8.6 \pm 0.73$ & \\
$25 \mathrm{OH}$ vit D $(\mathrm{ng} / \mathrm{ml})$ & Normal $(10-40)$ & & \\
& $38 \pm 7.78 ;$ & $14.4 \pm 6.8$ & \\
$1,25(\mathrm{OH}) 2$ & Normal (16-42) & & \\
vit D (pg/ml) & & & \\
& &
\end{tabular}

HypoCa TM, thalassemia major patients with calcium levels $<8.4 \mathrm{mg} / \mathrm{dl}$; NormoCa TM, thalassemia major patients with calcium levels $\geq 8.4 \mathrm{mg} / \mathrm{dl}$; $\mathrm{F}$, female; $\mathrm{M}$, male; $\mathrm{Hb}$, hemoglobin. 


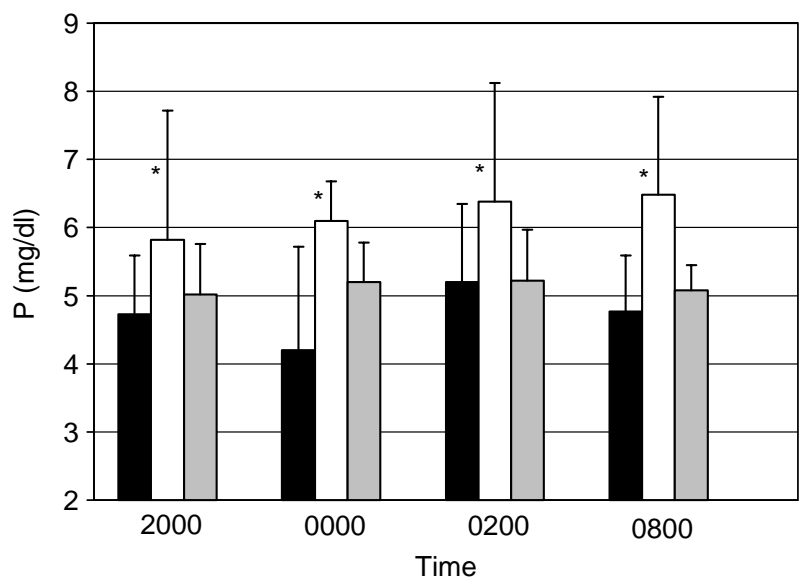

Figure 2 Nocturnal pattern of serum phosphorus $(P)(\mathrm{mg} / \mathrm{dl})$ in healthy controls (black bars), hypocalcemic TM (white bars) and normocalcemic TM patients (gray bars). Mean \pm S.D., ${ }^{\star} P<0.05$.

\section{Discussion}

Healthy subjects exhibit a diurnal variation of serum calcium and plasma PTH (1). Our study shows that measuring serum calcium levels at a single time point in the morning yields unreliable diagnosis of HPT, as previously suggested for other reasons (9). Nocturnal sampling, however, reveals both concealed and subclinical cases of hypoparathyroidism.

Given the well-documented delayed puberty in TM (6), one had to select either age or puberty for matching of the study and control groups. With regard to the known effect of puberty on mineral metabolism (8), we elected the latter option, with an obvious outcome of younger controls.

Modeling the hypothesis in TM, where the odds for developing HPT are high (11), enabled us to track HPT at its inception. A surprising finding was that every single one of our normocalcemic TM patients presented a certain degree of HPT, exceedingly more than the reported 4.5$30 \%(2-6)$. The possibility cannot be excluded that our patients are also less compliant with their chelating

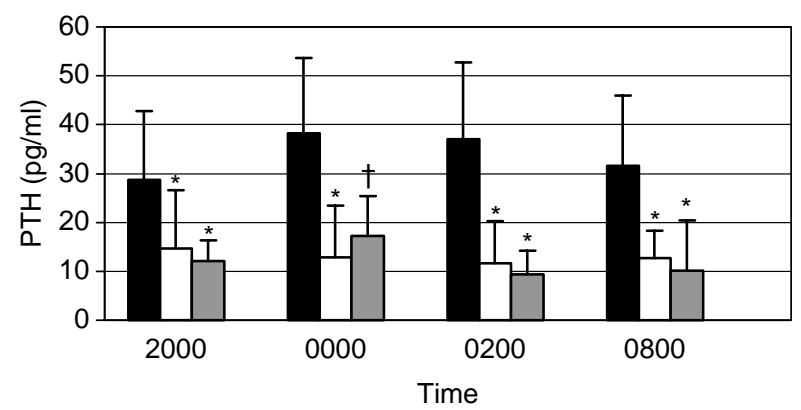

Figure 3 Nocturnal pattern of plasma parathyroid hormone (PTH) $(\mathrm{pg} / \mathrm{ml})$ in healthy controls (black bars), hypocalcemic TM (white bars) and normocalcemic TM patients (gray bars). Mean \pm s.D., ${ }^{\star} P<0.05,{ }^{\dagger} P<0.01$.

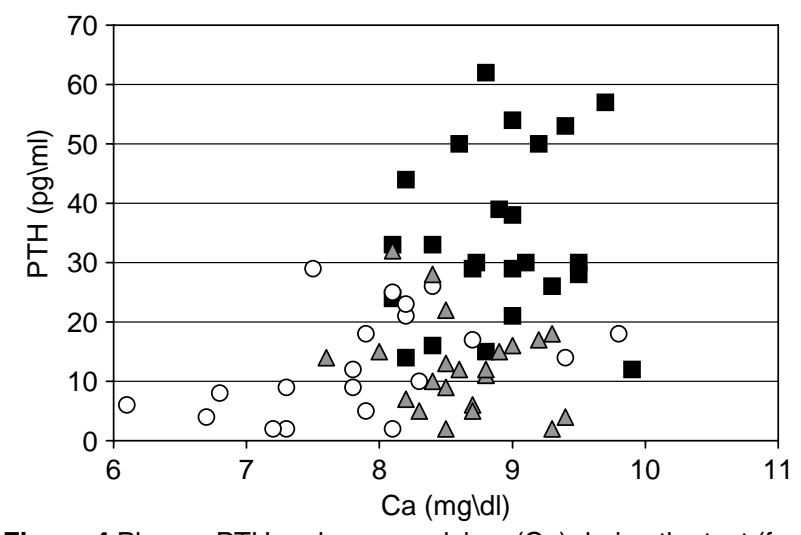

Figure 4 Plasma PTH and serum calcium $(\mathrm{Ca})$ during the test (four blood samples each at four time points) in healthy controls (black squares), hypocalcemic TM (white circles) and normocalcemic TM patients (gray triangles).

therapy, as evident from their abnormally high ferritin levels. TM patients of the present report also presented vitamin D insufficiency, as compared with the recommended pubertal levels (10). It is recommended that these very sick children, whose outdoors activities may be limited, should receive vitamin D supplementation.

Our data show two levels of potentially missed diagnosis, which we designate as concealed and subclinical HPT. The former is characterized by nocturnal hypocalcemia and the latter becomes apparent when plasma levels of PTH are plotted as a function of serum calcium levels, showing PTH insufficiency. TM patients with concealed HPT were older than those with a subclinical disease, indicating the progressive nature of HPT.

On a plot scale of all serum calcium against plasma PTH levels, the concept of concealed and subclinical HPT becomes evident. HPT shows decreases that are masked by the routine morning sampling, and may be unraveled by nocturnal sampling. Patients from the latter group show subclinical HPT with normocalcemia and relative PTH insufficiency. We recommend the periodic use of such measurements in all TM, and suggest that these tools may be applicable to other cases of suspected HPT.

\section{References}

1 el-Hajj Fuleiham G, Klerman EB, Brown EN, Choe Y, Brown EM \& Czeisler CA. The parathyroid hormone circadian rhythm is truly endogenous - a general clinical research center study. Journal of Clinical Endocrinology and Metabolism 197782 281-286.

2 De Sanctis V, Vullo C, Bagni B \& Chiccoli L. Hypoparathyroidism in beta-thalassemia major. Clinical and laboratory observation in 24 patients. Acta Hematological 199288 105-108.

3 Gabriele O. Hypoparathyroidism associated with thalassemia. South Medical Journal 197164 115-116.

4 Oberklaid F \& Seshadri R. Hypoparathyroidism and other endocrine dysfunction complicating thalassemia major. Medical Journal of Australia 19751 304-306. 
5 Pratico G, Di Gregorio F, Caltabiano L, Palano GM \& CarusoNicoletti M. Calcium phosphate metabolism in thalassemia. Pediatria Medica e Chirurgica 198820 265-268.

6 Tiosano D \& Hochberg Z. Endocrine complication of thalassemia. Journal Endocrinology Investment 200124 716-723.

7 Solberg HE. Establishment and use of reference values. In Tietz Textbook of Clinical Chemistry, edn 2, pp 454-484. Eds CA Buris \& ER Ashwood, Philadelphia, PA: WB Saunders, 1994.

8 Hochberg Z \& Tiosano D. Disorders of mineral metabolism. In Pediatric Endocrinology, Mechanisms, Manifestations and Management, pp 614-640. Eds O Pescovitz \& E Eugster, Philadelphia, PA: Lippincott Williams and Wilkins, 2004.

9 Ahmad AM, Hopkins MT, Fraser WD, Ooi CG, Durham BH \& Vora JP. Parathyroid hormone secretory pattern, circulating activity and effect on bone turnover in adult growth hormone deficiency. Bone 200332 170-179.

10 Hochberg Z, Bereket A, Davenport M, Delemarre-Van de Waal HA, De Schepper J, Levine MA, Shaw N, Schoenau E, Weisman Y \& Zadik Z. Consensus development for the supplementation of vitamin D in childhood and adolescence. Hormone Research 200258 39-51.

11 Angelopous NG, Goula A, Rombopoulos G, Kaltzidoy V, Kaounda E, Kaltsas D \& Tolis G. Hypoparathyroidism in transfusion-dependent patients with beta-thalassemia. Journal Bone Mineral Metabolism 200624 138-145.

Received 6 July 2006

Accepted 11 October 2006 